\title{
RADIATION DAMAGE IN SILICON PARTICLE DETECTORS IN HIGH LUMINOSITY EXPERIMENTS*
}

\author{
AgnieszKa Oblakowska-Mucha
}

for the RD50 Collaboration

AGH University of Science and Technology

Faculty of Physics and Applied Computer Science

Kraków, Poland

(Received October 3, 2017)

\begin{abstract}
Radiation damage is nowadays the most serious problem in silicon particle detectors placed in the very harsh radiation environment. This problem will be even more pronounced after the LHC Upgrade because of extremely strong particle fluences never encountered before. In this review, a few aspects of radiation damage in silicon trackers are presented. Among them, the change in the silicon lattice and its influence on the detector performance are discussed. Currently applied solutions and the new ideas for future experiments will be also shown. Most of the results presented in this summary were obtained within the RD50 Collaboration
\end{abstract}

DOI:10.5506/APhysPolB.48.1707

\section{Introduction}

Silicon detectors were successfully used in the LEP era for the vertices reconstruction and tracking of charge particles. Since the precision of impact parameter requires measurement of the first point of track close to the interaction point, the radiation damage gives the imminent threat to the silicon tracking detectors. The luminosity of the current LHC experiment reaches the level of $10^{34} \mathrm{~cm}^{-2} \mathrm{~s}^{-1}$. All presently operating silicon detectors will have to be replaced by the year 2025, during the main LHC Upgrade, when the luminosity will be risen by the order of magnitude. The accompanying enlargement of particle fluence enforced the quest for new structures and techniques more radiation tolerant than current solutions.

* Presented at the $2^{\text {nd }}$ Jagiellonian Symposium on Fundamental and Applied Subatomic Physics, Kraków, Poland, June 3-11, 2017. 


\section{Characterization of the radiation damage in silicon trackers}

Charged particles that propagate through the active volume of silicon lose their energy mainly by ionization. Simultaneously, both charged and neutral particles may also lose their energy through non-ionizing collision with the lattice atoms (silicon or dopant). That may cause the radiation damage i.e. the permanent changes in the semiconductor material. In the consequence of the collision, silicon atom can be displaced and it can install itself between regular lattice sites forming interstitials and vacancies (empty sites). If the knocked atom receives sufficient energy, it can travel through the crystal causing secondary defects, point or clusters, depending on its energy.

In order to describe the radiation damage in silicon bulk, a NIEL hypothesis is introduced. It assumes that any displacement damage in the lattice is linearly proportional to the amount of energy accumulated by the lattice [1]. Since displacement damage depends on the type of particle and its energy, it is customary to relate the damage to some reference particle (i.e., $1 \mathrm{MeV}$ neutron).

Most of the primary effects vanish since interstitials and vacancies can anneal, but also vast of defects either remain stable or may interact with the crystal impurities and cause the permanent changes. Among well-recognized defects are so-called A-centers (a combination of a vacancy and oxygen), E-centers (a vacancy-phosphorus complex) or divacancy (neighbor two missing silicon). The n-type silicon is usually doped with phosphorus and the presence of vacancy right to the donor dopant changes the electrical properties of the crystal and the phosphorus atom cannot fulfill the role of dopant anymore. This process is called "donor removal" and is very malignant for the silicon detector exposed to high particle radiation fluence. A great deal of defects created by the particle fluence above $10^{12} \mathrm{~cm}^{-2} n_{\text {eq }}$ are established and studied with proper methods of measurement and analysis which were developed within the RD50 Collaboration ${ }^{1}$, see, for example, [2].

The electrical properties of the created defects turn out to be more complicated than the initial semiconductor. Defect states can act as donors, acceptors (the dominant defect charge states), or can be electrically neutral. In all the cases, they have negative effect on detector performance:

- they can capture and emit electrons and holes (they act as recombination generation center),

- they can capture and emit electron with some delay. In this case, the signal comes too late for the efficient detection,

\footnotetext{
${ }^{1}$ RD50-Radiation hard semiconductor devices for very high luminosity colliders, http://rd50.web.cern.ch/rd50/
} 
- they can change the charge density in the active region thus requiring an increase of bias voltage for the full depletion.

Since the created defects can anneal, the impact of radiation damage is not constant after irradiation has stopped. Silicon interstitial and vacancies can recombine, stable defects are also able to transform and become inactive, what may be good for the sensor performance. On the other hand, inactive defects can also be reactivated and, thus, increase the impact of radiation damage yet again. The rate of damage is strongly dependent on temperature and can be predicted based on models such as the Hamburg model [1].

\subsection{Macroscopic changes due to the radiation}

Great effort has been made to correlate the macroscopic changes observed in silicon detectors with specific microscopic defects described in the previous section. In general, they can influence the sensor operation in three main ways:

- increase in leakage current, caused by the formation of mid-gap generation and recombination centers that facilitate the transition of electrons from the valence to conduction band. The increase is proportional to the particle fluence,

- change of the effective doping concentration of the sensor, which has an impact on the operating voltage needed for total depletion,

- loss of charge collection efficiency due to charge carrier trapping in defect states within the band gap. This effect causes a loss of signal, a main concern in heavily irradiated sensors.

Radiation-induced effects influence the silicon detector performance. The negative effects can cause progressive degradation of the detector. Having foreseen that, the study of new material and structures are currently undertaken.

\section{Development of radiation tolerant silicon detectors}

Semiconductor can be made more radiation-tolerant by deliberate setting the impurities during processing. These impurities interact with impinging radiation thus minimalizing its harmfulness. Thinner and segmented sensors are also considered as more radiation-tolerant since the damage is usually proportional to the sensor volume. Loss of signal charge can be avoided by reducing the collection time or distance to the electrodes. Decreasing charge may be regenerated by the process of multiplication in the high electric field 
in whole or only in thin layer of detector volume. In the following chapter, selected solutions towards the more radiation-tolerant silicon detector are presented. These structures are currently developed within the RD50 Collaboration for the HL-LHC and future experiments.

\section{1. $3 D$ pixels}

3D pixel structures have electrodes etched as long columns penetrating the silicon substrate, see Fig. 1. This technology, proposed two decades ago [3], has been recently applied in two LHC detectors - ATLAS IBL and AFP.
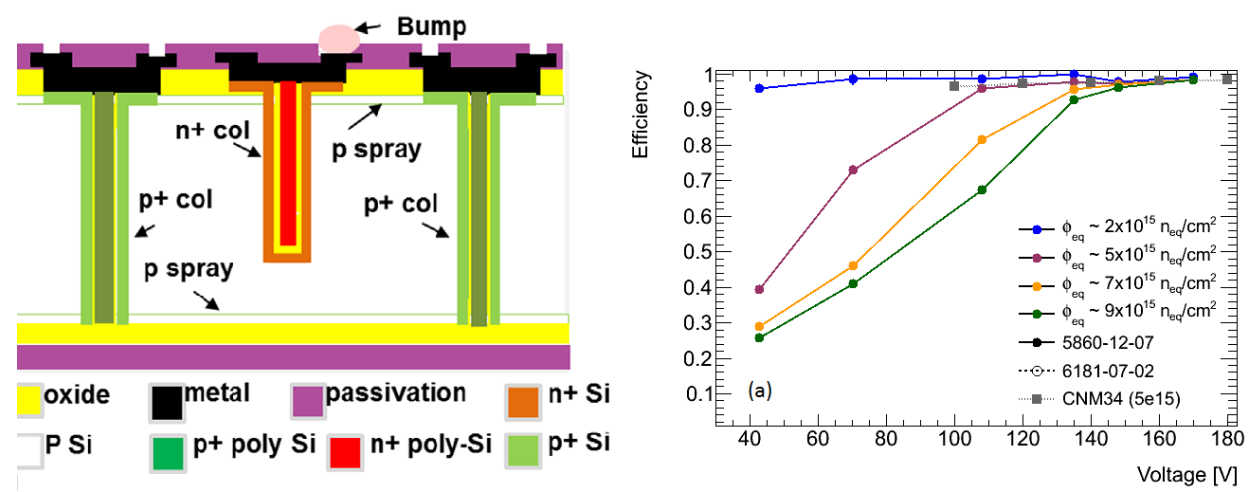

Fig. 1. The schematic view of double sided pixel sensor (left). Pixel efficiency versus bias voltage for IBL type 3D pixel sensors (right) [4].

In 3D sensors, the interelectrode distance can be made very small (less than $50 \mu \mathrm{m}$ ) without thinning the sensor. This reduces the collection distance without affecting the signal amplitude. The shorter distance means lower trapping probability after irradiation, faster response time, and lower depletion voltage. These factors make the $3 \mathrm{D}$ structures very radiation resistant. Two observed problems of this geometry: loss of efficiency in regions between electrodes and non-uniform spatial response may be partially overcome by tilting of the sensors and by improving the trenching technology respectively. One of the most important drawbacks of this structure is very sophisticated and expensive fabrication technique. Because of that, 3D structures practically may be used in smaller detectors, close to the IP.

First irradiation test performed on $3 \mathrm{D}$ pixels showed that the signal efficiency is about $60-70 \%$ at the fluence of $5 \times 10^{15} \mathrm{~cm}^{-2} n_{\mathrm{eq}}$ and despite it goes down to $30 \%$ at about $10^{16} \mathrm{~cm}^{-2} n_{\text {eq }}$, the induced signal is still about 6000 electrons and the efficiency can be improved with small increase of bias voltage, see Fig. 1 [4]. 
For the purpose of high luminosity experiment, within the RD50 Collaboration, a joint project of ATLAS, CMS and the LHCb is underway. The main objectives are production of smaller area 3D pixel on thin sensors.

\section{2. $H V-C M O S$}

The HV-CMOS pixel sensor operating concept is shown in Fig. 2. The sensor has a structure of three wells: a lightly doped deep n-well is implanted in a low low-resistivity p-type substrate and plays the role of collecting electrode. The $\mathrm{p}-\mathrm{n}$ junction is formed between the $\mathrm{n}$-well and $\mathrm{p}$-type substrate and is partially depleted by applying a reversed bias voltage. The electronhole pairs generated by charged particles passing through the depleted region are separated and quickly collected by drift in high electric field. The fast charge collection time reduces charge loss due to trapping and in that way improves the radiation tolerance of HV-CMOS sensors. The HV-CMOS technology requires the biasing voltage lower than $100 \mathrm{~V}$, the active layer (depletion zone) is thin (about 10-20 $\mu \mathrm{m}$ ) what corresponds to about 1000 electron-hole pairs for minimum ionizing particles (MIPs).
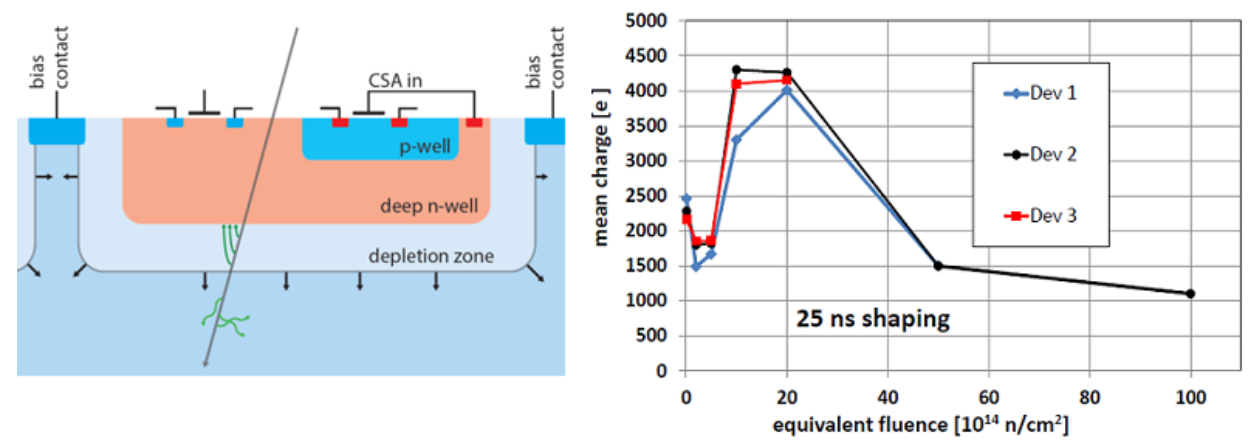

Fig. 2. The cross section of the HV-CMOS sensor (left) [4] . Collected charge versus fluence for three values of the bias voltage for AMS $350 \mathrm{~nm}$ production (right) [5].

The entire CMOS electronics is implemented in the deep n-well avoiding the expensive bump bonding. Due to the industrial production, the cost of production is relatively low and HV-CMOS structures can be used on large scale.

The radiation tolerance of HV-CMOS sensors have been studied as one of the project of the RD50 Collaboration. Two main structures were used: $350 \mathrm{~nm}$ and $180 \mathrm{~nm}$ AMS sensors with CHESS-1 and FE-I4 front-end readout chips, respectively. Sensors were irradiated to very high neutron fluences up to $2 \times 10^{16} \mathrm{~cm}^{-2} n_{\text {eq }}[5,6]$. The behavior of the collected charge as a function of accumulated fluence is presented in Fig. 2. The first drop of the observed 
collected charge occurs at a fluence corresponding to $10^{15} \mathrm{~cm}^{-2} n_{\mathrm{eq}}$ and is caused by the charge trapping mainly of the diffusion component. For higher fluences, the collected charge is rising since the active volume of the sensor (the depleted region) is increasing (acceptor removal). This phenomenon stops at about of $2 \times 10^{15} \mathrm{~cm}^{-2} n_{\mathrm{eq}}$ when the formation of new defects and space charge invokes intense charge trapping. Albeit, charge collection is decreasing, it reaches about $90 \%$ of the initial signal even after irradiation with fluence $2 \times 10^{16} \mathrm{~cm}^{-2} n_{\mathrm{eq}}$, see Fig. 2 [6]. This result, together with the relatively low cost and industrial production method and good physics performance, makes the HV-CMOS technology very promising for use in high-radiation environments.

\section{Edge-TCT}

Edge Transient Charge Technique (Edge-TCT) is a method of reconstruction the electric field in silicon sensors [7]. A red or infrared laser is used for the induction of the signal by ionization of the sensor bulk. Strong laser pulses are directed towards the detector edge, perpendicular to the strips. Scans across the detector thickness enables measurement of the induced current at a given depth and then - drift velocity, thus the electric filed, and charge collection profiles.
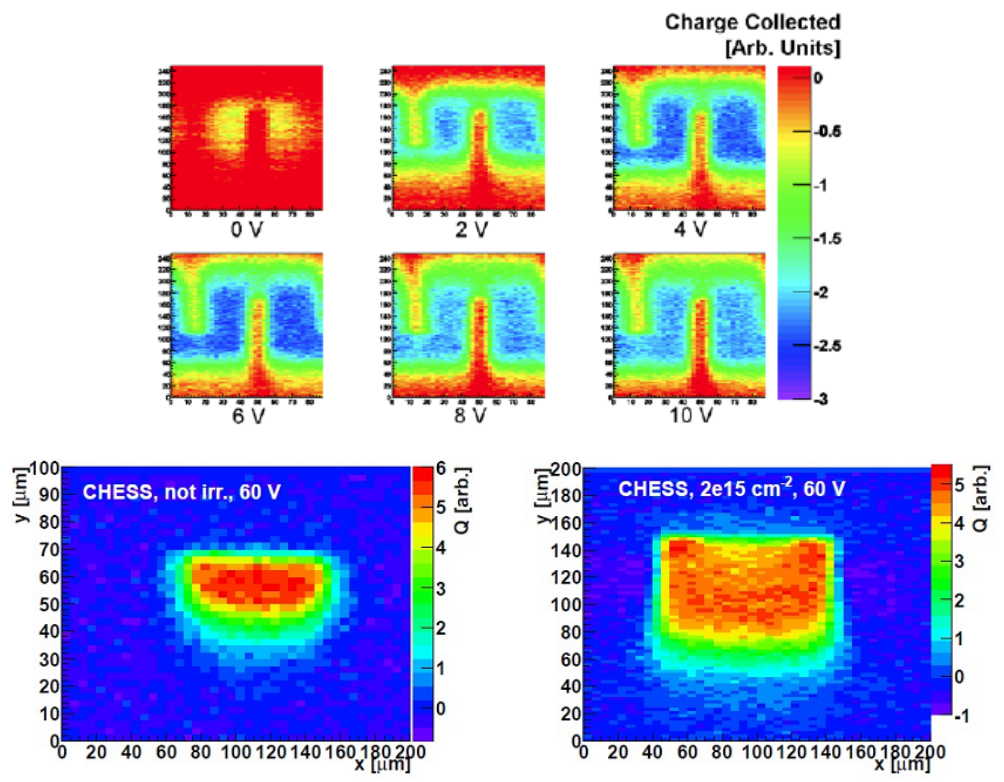

Fig. 3. Charge collection maps using Edge-TCT for 3D Pixels. The electric field is growing while increasing the bias voltage (left) [7]. The charge profiles for HVCMOS CHESS-1 sensor before and after irradiation (right) [4]. 
This remarkable method is commonly used for study substrate properties before and after irradiation at different bias voltage. In Fig. 3 the examples of some Edge-TCT results are shown: charge collected in 3D pixels and depleted zone in HV-CMOS before and after irradiation. In all the cases, Edge-TCT method gauges the electric field with high spatial resolution determining this way whether the sensor is fully depleted. From the charge profiles, it can also resolve the composition of the measured current (drift or diffusion, electron or holes) providing valuable information on the processes that occurred after irradiation (donor and acceptor removal, etc.).

\section{Summary}

The silicon detectors that are currently taking data were designed for ten years of operation. The main reason for replacement is gradually worsening of the performance caused by severe particle radiation. Existing technologies, such as planar strip and pixel silicon sensors will be operating until the year 2023 exploiting the physics program of the LHC, but they cannot withstand the fluence at the level of $2 \times 10^{16} \mathrm{~cm}^{-2} n_{\text {eq }}$, predicted for the detector inner layers at HL-LHC. Thus, an extensive program has been taken by the RD50 Collaboration to study the origin of created defects, develop radiation hard structures and materials, and work out solutions to mitigate the harmful radiation impact. Among proposed and recommended solution are 3D pixels and HV-CMOS. 3D structures incorporate all beneficial features: good resolution for physics studies, sufficient radiation hardness and relatively low depletion voltage. The main obstacle against wide usage is complicated and expensive fabrication. Contrary HV-CMOS - the industrial processing and good radiation tolerance pave the way for future usage at large scale. None of the results could be obtained without a method of measurement showing the outcome of modernization. With the use of EdgeTCT, the properties of semiconductor material before and after irradiation can be characterized.

I would like to express my gratitude to the National Science Centre, Poland (NCN) for financial support under the contract No. UMO-2016/21/B /ST/01083.

\section{REFERENCES}

[1] M. Moll, Thesis, Universitat Hamburg, 1999, DESY-THESIS-1999-040.

[2] I. Pintilie et al., Nucl. Instrum. Methods Phys. Res. A 611, 52 (2009). 
[3] S.I. Parker, C.J. Kenney, J. Segal, Nucl. Instrum. Methods Phys. Res. A 395, 328 (1997).

[4] J. Lange et al., JINST 11, C11024 (2016).

[5] A. Affolder et al., JINST 11, P04007 (2016).

[6] M. Fernandez Garcia et al., JINST 11, P02016 (2016).

[7] G. Stewart et al., JINST 8, P03002 (2013). 